Chain ordering of hybrid lipids can stabilize domains in saturated/hybrid/cholesterol lipid membranes

This article has been downloaded from IOPscience. Please scroll down to see the full text article.

2010 EPL 9128002

(http://iopscience.iop.org/0295-5075/91/2/28002)

View the table of contents for this issue, or go to the journal homepage for more

Download details:

IP Address: 131.215.220.185

The article was downloaded on 21/10/2010 at 16:46

Please note that terms and conditions apply. 


\title{
Chain ordering of hybrid lipids can stabilize domains in saturated/hybrid/cholesterol lipid membranes
}

\author{
T. Yamamoto ${ }^{1(a)}$, R. Brewster ${ }^{1,2}$ and S. A. Safran ${ }^{1}$ \\ ${ }^{1}$ Department of Materials and Interfaces, Weizmann Institute of Science - Rehovot, Israel, 76100 \\ 2 Department of Applied Physics, CalTech - Pasadena, CA, USA
}

received 26 May 2010; accepted in final form 8 July 2010

published online 4 August 2010
PACS 82.70.Uv - Surfactants, micellar solutions, vesicles, lamellae, amphiphilic systems (hydrophilic and hydrophobic interactions)
PACS 64.75.Yz - Self-assembly
PACS 87.16.D- - Membranes, bilayers, and vesicles

\begin{abstract}
We use a liquid-crystal model to predict that hybrid lipids (lipids that have one saturated and one unsaturated tail) can stabilize line interfaces between domains in mixed membranes of saturated lipids, hybrid lipids, and cholesterol (SHC membranes). The model predicts the phase separation of SHC membranes with both parabolic and loop binodals depending on the cholesterol concentration, modeled via an effective pressure. In some cases, the hybrid lipids can reduce the line tension to zero in SHC membranes at temperatures that approach the critical temperature as the pressure is increased. The differences in the hybrid saturated tail conformational order in bulk and at the interface are responsible for the reduction of the line tension.
\end{abstract}

Copyright (C) EPLA, 2010

Introduction. - The lipid raft hypothesis suggests that nanoscopic domains in biological membranes play an important role in a range of cellular functions $[1,2]$. Fluorescence microscopy studies show that membranes, comprised of saturated lipids with two saturated tails, unsaturated lipids with two (partially) unsaturated tails, and cholesterol (SUC membranes) exhibit phase separation into domains of liquid-disordered phase $\left(L_{d}\right.$ phase) that coexist with domains of liquid-ordered phase $\left(L_{o}\right.$ phase) [3]. The $L_{d}$ phase is rich in unsaturated lipids and the tails of the lipids are disordered, while the $L_{o}$ phase is rich in saturated lipids and cholesterol and the tails of the lipids are more ordered [4]. The lipid raft hypothesis in biological membranes envisages domains on the order of 10-100 $\mathrm{nm}$ that are stable or metastable [2]. However, as is usually the case in macroscopic phase separation, domains in model SUC membranes exhibit coarsening due to the positive line tension and their size eventually reaches the order of the system size, i.e. 1-10 $\mu \mathrm{m}$ [3].

One reason for the discrepancy in the domain size between model SUC and biological membranes may be that lipids with two unsaturated tails are only minor components of biological membranes and not commonly found. On the other hands, lipids with one saturated

\footnotetext{
(a) E-mail: tetsujava@hotmail.com
}

and one unsaturated tail, e.g. POPC or SOPC, here termed "hybrid" lipids are major components of biological membranes $[5,6]$. High-resolution techniques, e.g. fluorescence resonance energy transfer $[7,8]$ and antibody reporters [9], suggest that membranes containing saturated lipids, hybrid lipids, and cholesterol (SHC membranes) show nanoscopic domains in some regions of their phase diagrams. However, this is somewhat controversial, since coarsening domains were also observed in SHC membranes by fluorescence microscopy [10]. It is possible that nanoscopic domains are only stable in a limited part of the phase diagram and/or nanoscopic domains are only metastable. This motivated us to theoretically study the phase separation and the line tension between domains in SHC membranes.

The chain packing theory $[11,12]$ suggests that the chain packing incompatibility between the tails of saturated lipids and the permanently kinked tails of unsaturated lipids drives the phase separation in SUC membranes when the tails of saturated lipids are relatively ordered. This implies that chain packing incompatibility at the interface gives rise to the positive line tension between domains in this membrane. A positive line tension drives domain coarsening, thus, finite-size domains can only be stable if the line tension is reduced to zero; the finite size is then determined by packing considerations at the interface [13]. 
Since hybrid lipids have both saturated and unsaturated tails, they may reconcile the chain packing incompatibility at the interface and reduce the line tension. Our previous calculations used a phenomenological theory to demonstrate that small quantities of hybrid lipids added to SUC membranes that exhibit phase separation can reduce the line tension between domains to zero [14] and stabilize nanoscopic domains whose size is then fixed by the spontaneous curvature [13], in analogy with the stabilization of microemulsions by surfactants [15]. However, in SUC membranes, zero line tensions are achieved only at low temperatures far from the critical point due to the mixing entropy cost to localize the hybrid lipids to the interface.

It is well known that oil-surfactant-water three-body interactions are responsible for the reduction of the interfacial tension at oil-water interfaces and stabilize microemulsions [16]. How can hybrid lipids stabilize nanoscopic domains in SHC membranes where saturatedhybrid-unsaturated three-body interactions are absent? In our previous study [14], the chain order was not taken into account explicitly. However, the conformation of the tails of hybrid lipids depends on their location and hybrid lipids behave differently in the bulk and at the interface reflecting the difference in chain conformational states: in the bulk hybrid-rich phase, there are many unsaturated tails (of the hybrid lipids) whose packing is incompatible with the ordered saturated tails (of either the saturated or the hybrid lipids), and the saturated tails of the hybrid lipids are in their disordered state. However, at the interface with the saturated-rich phase, the saturated tails of the hybrid lipids are relatively ordered due to their proximity to the saturated-rich phase that contains ordered saturated tails. This maintains the driving force for phase separation in the bulk and provides the effective three-body interactions (between saturated lipids, the ordered hybrid lipids at the interface, and the disordered hybrid lipids in the bulk) that are responsible for the reduction of the line tension. Indeed, domains in SHC membranes may achieve zero line tension even at relatively high temperatures near the critical point since hybrid lipids are already at the interface in contrast to the hybrid lipids in SUC membranes, where hybrid lipids lose mixing entropy by being localized at the interface [14]. In this letter, we used a liquid-crystal model [17] to demonstrate that hybrid lipids in SHC membranes can achieve low or zero line tension between domains in SHC membranes at relatively high temperature close to the critical point.

Liquid-crystal model. - Since chain packing incompatibility drives the phase separations of SHC membranes and results in a positive line tension between domains, we must take into account the ordering of the lipid chains in a simple manner. In chain packing theories, the hydrophobic cores of homogeneous membranes are often modeled as isotropic liquids of alkane [18]. However, we focus on the fact that the hybrid lipids can modify its chain conformational order depending on its environment. We therefore
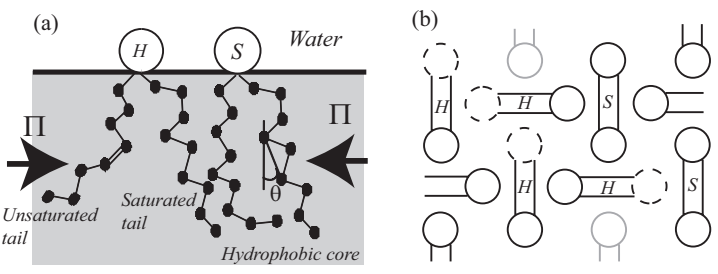

Fig. 1: Side (a) and top (b) views of an SHC membrane. $\theta$ is the angle between the membrane normal and the chain segment and the order of the saturated tail is characterized by the chain order parameter $S_{s}$. The lateral pressure $\Pi$ (which, in part, arises from the condensing effect of cholesterol) is applied to the hydrophobic core. Solid and dashed circles in (b) represent saturated and unsaturated tails, respectively. The two circles represent the tails of a lipid shown as dumbbell. The lipid tail interacts with nearest neighbors except for the other tail of the same lipid.

model the hydrophobic core of mixed membranes as a liquid crystal of alkane. Similar analogies with liquid crystals have been used to analyze the main phase transitions $[19,20]$ and the mechanical properties [21,22] of homogeneous membranes. Since the phase separations into $L_{d}$ and $L_{o}$ domains are driven by both lipid composition and temperature, our model regards mixed membranes as liquid crystals with both lyotropic and thermotropic properties.

In this picture, the ordering of saturated tails is characterized by the nematic order parameter $S_{s} \equiv \int \mathrm{d}(\cos \theta) \frac{3 \cos ^{2} \theta-1}{2} n(\cos \theta)$, where $\theta$ is the average angle between the chain segments and the membrane normal, see fig. $1(\mathrm{a})$, and $n(\cos \theta)$ is the single chain distribution function with respect to $\theta$ [23]. Lipid tails in the hydrophobic core pack to minimize their contact with water, i.e. surface tension. This surface tension compresses the hydrophobic core until it is balanced (for a tensionless membrane) with the pressure due to the excluded-volume interactions between chains as well as with the polar head group interactions $[15,18]$. The lateral pressure $\Pi$ represents the compressive force exerted to the hydrophobic core by this effect. We take into account the lateral pressure in an extension of the Maier-Saupe theory $[23,24]$ of liquid crystals, used to treat 3D binary nematic mixtures [24] to predict both the bulk and interfacial properties of SHC membranes. In general, the local chain order parameters and the lateral pressure vary across the finite thickness of the membrane $[15,18]$. In our $2 \mathrm{D}$ model, we define $S_{s}$ and $\Pi$ as the mean values of the chain order parameter and the lateral pressure over the thickness of hydrophobic core, respectively. The details of the liquid-crystal model and its prediction will be published separately [17].

We model the hydrophobic core of the membrane as a $2 \mathrm{D}$ face-centered square lattice that is fully populated with the tails of saturated and hybrid lipids, see fig. 1. Mixtures of cholesterols and lipids are known to occupy less area than expected from the sum of the separated constituents (condensing effect) [25], probably because 
cholesterol forms complexes $[25,26]$ or nano-crystals with lipids via saturated tails [27]. Cholesterol packs lipid tails more closely and therefore increases the lateral pressure arising from the thermal motions of the chains. In our model, we implicitly take into account the cholesterol by changes in the lateral pressure in the membrane. Thus, the total lateral pressure is the intrinsic pressure due to the lipid chains and polar heads at the interface and the excess pressure arising from the condensing effect of cholesterol. This approach is used to predict behaviors of cholesterol known from experiments in ref. [17].

Since the hydrophobic core of the membrane is in the liquid state, the optimal packing of the lipid chains is determined by the minimization of the free energy that includes the conformational entropy and the cohesive interactions. The efficient packing of hybrid lipids is hindered by their unsaturated tails, which are permanently kinked due to the cis double bond. However, the intermolecular interactions between the unsaturated tails of the hybrid lipids and the saturated tails of other lipids are reduced by the permanent kink only when the saturated tails of the opposing lipids are ordered. Thus, for simplicity, we neglect the interactions that do not depend on the chain order parameter $S_{s}$.

The objective of this paper is to demonstrate that hybrid lipids can adjust its internal degrees of freedom (i.e., the conformations of its saturated chain) to reduce the line tension. This concept is best illustrated in a simple model with a minimal number of parameters. We thus consider a model in which the saturated chain of the hybrid is identical to that of the saturated lipid and the unsaturated tails of the hybrid lipids are completely disordered. For simplicity, we represent the saturated chain conformation (for either the saturated and hybrid lipids) by a single order parameter $S_{s}$. This model predicts that the chain ordering transition temperature of a pure hybrid lipid membrane is half of the main transition temperature of a pure saturated lipid membrane. However, since our focus is on the generic interfacial properties of SHC membranes, we use this minimal model; accurate predictions of the bulk phase diagrams require several additional parameters (such as the chain lengths and characteristics of the double-bond regions) and are beyond the scope of this paper.

With this lattice model, the Gibbs free-energy density of the bulk is:

$$
\begin{aligned}
f_{\mathrm{bulk}}= & T[\psi \log \psi+(1-\psi) \log (1-\psi)] \\
& -\frac{1}{2} J_{\mathrm{ss}} S_{s}^{2}(1+\psi)^{2} \\
& +f_{\mathrm{conf}}(1+\psi)-\Pi S_{s}(1+\psi),
\end{aligned}
$$

where $\psi$ is the local concentration of saturated lipids and $S_{s}$ is the chain order parameter of the saturated tails of either the hybrid or saturated lipid. $T$ is the absolute temperature in units of the Boltzmann constant. $J_{\mathrm{ss}}$ is the excess interaction energy of two neighboring saturated tails by chain ordering. $f_{\text {conf }} \equiv T \int \mathrm{d}(\cos \theta)$ $n(\cos \theta) \log n(\cos \theta)$ is the free energy per tail arising from the entropy loss of ordered saturated tails [23]. The minimization of the free energy with respect to $n(\cos \theta)$ leads the chain distribution function at the equilibrium. $\Pi$ is the (rescaled) lateral pressure. The factor of $1+\psi$ in the last three terms of eq. (1) originates from the fact that saturated lipids carry two saturated tails while hybrid lipids carry only one saturated tail, i.e. $(2 \times \psi)+(1 \times(1-\psi))$. The last term of eq. (1) is the work done by the lateral pressure $\Pi$. The pressure results in a decrease of the area per tail by an amount proportional to $-S_{s} a_{0}^{2}$ compared with the area $a_{0}^{2}$ occupied by a completely disordered tail. It is important to note that in our model, if the saturated tails are not ordered, there are no net interactions that would give rise to phase separation. This highlights the role of chain ordering in promoting phase separation in SHC membranes.

The interfacial free energy, $g_{\mathrm{int}}$, arises from the gradient of the concentration that is large near the interface:

$$
\begin{aligned}
g_{\mathrm{int}}= & \frac{1}{8} J_{\mathrm{ss}} a_{0}^{2}\left[\nabla\left(S_{s}(1+\psi)\right)\right]^{2}+g_{\mathrm{ori}}(1-\psi) \\
& -\frac{1}{2} J_{\mathrm{ss}} S_{s}(1-\psi) a_{0} \sigma \cdot \nabla\left(S_{s}(1+\psi)\right),
\end{aligned}
$$

$\sigma=\int \mathrm{d} \phi(\cos \phi, \sin \phi) \rho(\phi)$ denotes the average orientation of the $2 \mathrm{D}$ vector joining the centers of mass of the saturated and unsaturated tails of a given hybrid lipid, where $\rho(\phi)$ is the orientational distribution with respect to the orientational angle $\phi$. For our choice of the face-centered square lattice, the bulk binary nearest-neighbor interactions are independent of $\sigma$ and the hybrid orientation is important only near the interface, see fig. 1 . The first term in eq. (2) is the gradient energy [15]. The next term, $g_{\text {ori }} \equiv T \int \mathrm{d} \phi \rho(\phi) \log \rho(\phi)$, is the free energy per hybrid lipid arising from the orientational entropy. The third term is calculated from the energetics of the lattice model. It is analogous to the electrostatic energy stored by a dipole gas in an electric field. Just as dipoles lower their energies by orienting towards electric fields, hybrid lipids lower their energies by orienting their saturated tails in the direction of the concentration gradient: i.e. towards $L_{o}$ domains. The minimization of the free energy with respect to $\rho(\phi)$ leads the orientational distribution function in the equilibrium. Although we determined the prefactors of the interaction terms in eqs. (1) and (2) from the lattice model, the line tension is only sensitive to the ratio between the prefactors of the first and the third terms of this equation.

Phase diagram. - The minimization of the bulk free energy, eq. (1), with respect to $n(\cos \theta)$ yields the equilibrium single chain distribution function that is now a function only of the temperature $T$ and lipid concentration $\psi$. The bulk free energy is then written as a function of $T, \psi$ by using the equilibrium chain distribution function. We use the common tangent construction [15] to derive the binodal curves; the lipid concentrations in the two coexisting phases are obtained by the equilibrium condition of chemical potential and osmotic pressure. The 

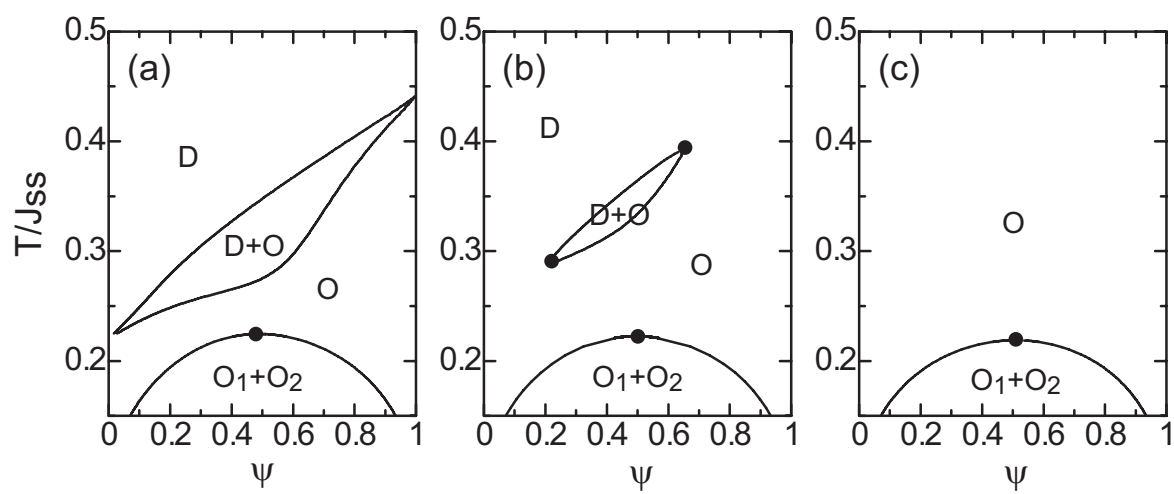

Fig. 2: The miscibility phase diagram is calculated numerically from eq. (1). (a)-(c) are the diagrams calculated for $\beta \Pi=0.0001$, 0.09, and 5.0, and provide examples of Regimes 1, 2, and 3, respectively, as discussed in the text. $D$ and $O$ indicate disordered and ordered phases, respectively. The critical points are marked by dots.

model predicts three, generic types of phase diagrams depending on the pressure, see fig. 2.

For small $\Pi$, there is a loop binodal without critical points at high temperatures and a parabolic binodal with a critical point at lower temperatures (Regime 1), fig. 2(a). Phase diagrams similar to fig. 2(a) were predicted by a phenomenological model for binary system [26]. The loop binodal extends to the two points where a membrane of pure saturated, $\psi=1$, or pure hybrid, $\psi=0$, exhibits ordering transitions. The phase separations in the loop binodal are associated with the (nematic) ordering transitions. The phase separations in the loop binodals are characterized by relatively large differences in the chain order but by relatively small difference in the lipid concentrations in the two separated phases (i.e., along the tie lines). The jump of the chain order parameter $S_{s}$ at the first-order ordering transition, which represents the main transition of lipid membranes, is responsible for the large differences in the chain order parameter $S_{s}$ in the twophase region. This jump decreases with increasing lateral pressure until the ordering transitions are suppressed in Regime 2. In the parabolic binodal, the membrane exhibits phase separation because the temperature is low enough so that the saturated tails of both the hybrid and the saturated lipids are ordered. This phase separation is characterized by relatively large differences in the lipid compositions but smaller differences in the chain order between the two separated phases. In the low-pressure regime, the phase separation is not driven by lateral pressure but by temperature.

For values of lateral pressure $\Pi$ slightly larger than that of a first threshold value, there is a narrow range in which the loop binodal exhibits critical points at its upper and lower ends (Regime 2), fig. 2(b). In this regime, the ordering transitions are suppressed by the pressure $\Pi$. However, the loop binodal remains in the phase diagram due to the presence of a temperature, at which the susceptibility $\partial S_{s} / \partial \psi$ is relatively large. This large value of susceptibility is a residue of the ordering transitions in Regime 1. The nature of the loop and parabolic binodals with regard to the chain order and lipid compositions are roughly the same as the corresponding binodals in Regime 1. The critical temperature and concentration of the upper critical points decrease, whereas those of the lower critical points increase with increasing $\Pi$. Eventually, for a value of the lateral pressure, $\Pi$, that is larger than a second threshold value, the loop binodal disappears because the susceptibility is no longer large (Regime 3), fig. 2(c). In this regime, the phase separations in the parabolic binodal are mostly driven by the pressure.

Line tension. - The concentration $\psi$, the chain order parameter $S_{s}$, and the orientational order parameter $\sigma$ at the interfaces are derived by minimizing the total free energies, i.e. $g_{\mathrm{bulk}}+g_{\mathrm{int}}$ with respect to $\rho(\phi), \psi$, and $n(\cos \theta)$ with $g_{b u l k}=f_{\text {bulk }}-\mu \psi(z)$ where $\mu$ is the chemical potential. The line tension $\lambda$ is calculated from those parameters by $[14,15]: \lambda=\int \mathrm{d} z\left(g_{\text {bulk }}(\psi(z))+g_{\text {int }}-\right.$ $\left.g_{\text {bulk }}(\psi(\infty))\right) . g_{\text {bulk }}(\psi(\infty))$ is the thermodynamic potentials in the bulk that is equal in each of the coexisting domains due to the equilibrium of the osmotic pressure.

The line tension is calculated numerically for the interfaces that separate bulk phases whose equilibrium is determined by the parabolic binodals and is plotted as a function of temperature for several values of the pressure, $\Pi$, in fig. 3. While for small $\Pi$, it is necessary to decrease the temperature to below $30 \%$ of the critical temperature to reduce the line tension to zero, for higher-pressure values, the line tension goes to zero for temperatures just a little below the critical temperature. This pressure dependence suggests that one might experimentally observe the line tension reduction by hybrid lipids for relatively high cholesterol concentrations, since the condensing effect of cholesterol corresponds to increasing $\Pi$ in our model.

The line tension near the critical temperature $T_{c}$ is calculated from our model by a Ginzburg-Landau expansion [15] as

$$
\lambda \simeq \lambda_{0} \sqrt{1-\frac{1}{2} \frac{J_{\mathrm{ss}}}{T} S_{c}^{2}\left(1-\psi_{c}\right)},
$$

where $S_{c}$ is the order parameter at the critical point. $\lambda_{0}$ is the line tension without the orientational contributions of 


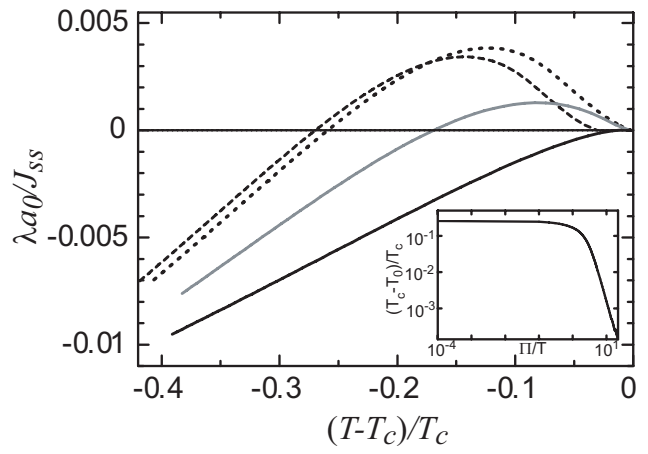

Fig. 3: The line tensions $\lambda$ calculated numerically as functions of the temperature $T$ for $\Pi / T=0.0001$ (dotted line), 0.09 (dashed line), 1.0 (gray line), and 5.0 (solid line). Inset: the temperature $T_{0}$ where zero line tension is achieved $v s$. pressure $\Pi / T$ (log-log plot).

the third term of eq. (2) and scales with temperature as $\left(\left(T_{c}-T\right) / T_{c}\right)^{3 / 2}[15]$. The hybrid lipids at the interfaces reduce the line tension from $\lambda_{0}$ by orienting and ordering their saturated tails in the vicinity of the interface between the hybrid-rich and saturated-rich phases. The reduction of the line tension is larger when the order parameter $S_{c}$ of the hybrid lipids is larger. However, $S_{c}$ is not large enough to reduce the line tension to zero near the upper and lower critical points of the loop binodals in Regime 2. On the other hand, near the critical points of parabolic binodals, $S_{c}$ is sufficiently large, $0.8-1.0$, so that the argument of the square root in eq. (3) can be small enough to decrease the line tension to zero when the temperature is lowered from $T_{c}$ by $\Delta T \leqslant T_{c}-\frac{1}{2} J_{\mathrm{ss}} S_{c}^{2}\left(1-\psi_{c}\right)$. The chain ordering of the hybrid lipids near the interface causes the line tension to be reduced to zero even at temperatures somewhat below the critical temperature. This situation is very different from the role of hybrid lipids in SUC membranes, where the reduction of line tension by hybrid lipids is strongly suppressed by the mixing entropy of the hybrid lipids.

The reason why the line tension reduction is more effective and can occur at temperatures just a little below the critical temperature in SHC membranes is because the hybrid lipids are already at the interface; this is in contrast to hybrid lipids in SUC membranes which lose mixing entropy when they are localized at the interface. The surfactant-like action in SUC membranes can only occur when the mixing entropy cost is negligible. Therefore, the line tension reduction is predicted to be strong only at relatively low temperatures. In SHC membranes, the chains of the hybrid lipids near the interface are predicted to be more ordered than in the bulk. The hybrid lipids reduce the line tension by ordering their chain conformations and orientations at the interfaces rather than by acting as surfactants; these relatively ordered states can be stable even close to the critical temperature, at least at high pressures within our model.

Discussion. - We have demonstrated that the degree of freedom of the hybrid lipids to order their chains in the vicinity of the interface can, in some cases, reduce the line tension to negative values even relatively close to (but below) the critical point. At first glance it appears that the existence of a negative line tension tends to increase the total length of the interface without bound, thereby returing the membrane to the mixed state. However, as we have shown [13], hybrid lipids provide a mechanism to prevent the domains from becoming too small in size. The projected areas of the saturated and unsaturated tails of hybrid lipids are different due to the differences in their chain ordering. In ref. [13] we used packing arguments to demonstrate that this difference in the projected area gives rise to $1 \mathrm{D}$ spontaneous curvature that sets the domain size in a certain range. Since there is always a temperature region where the SHC membranes show macroscopic phase separation at immediate vicinity of the critical point, see fig. 3, SHC membranes should show the usual critical scattering of small wave vectors [15]. There is a transition from macroscopic to microphase separation of the order of $10-100 \mathrm{~nm}$ with decreasing the temperature [13].

The presence of nanoscopic domains in SHC membanes was suggested experimentally by high-resolution techniques [7-9]. However, this is somewhat controversial since microscopic studies on SHC membranes (e.g., sphingomyeline/POPC/cholesterol) reported macroscopic domains [10]. Our theory predicts that the phase separations in SHC membranes can change from macroscopic to nanoscopic as the lateral pressure is increased. Experimentally, this corresponds to increasing the concentration of cholesterol. Thus, SHC membranes may exhibit nanoscopic domains in only a portion of their ternary phase diagram; where the domains are macroscopic and where they are nanoscopic (corresponding to the regime of negative line tension) may be a strong function of temperature, the cholesterol concentration and the overall lipid chains lengths. Another possibility is that the line tension is still positive under the conditions of the fluorescence experiments, but that the 1D spontantous curvature maintains the nanoscopic domains in a metastable state. Further systematic experiments are necessary to clarify this issue. We also note that these predictions are applicable to SHC membranes in which chain packing incompatibility plays a major role in the phase separations. The contributions of other lipid properties, e.g. head interactions and chain lengths will be included in a future study.

We have demonstrated that the line tension is reduced to zero for the parabolic binodal. This binodal corresponds to a system in which both of the coexisting domains are in the $L_{o}$ phase, but have different lipid compositions since both of the bulk phases exhibit relatively large chain order parameters. The loop binodal in Regime 2 has critical points and is characterized by relatively large differences of chain order parameters in the coexisting bulk phases. Thus, this binodal predicts phase separation into $L_{d}$ phase domains that coexist with $L_{o}$ phase domains. However, the model presented does not predict zero line tension of the interfaces that arise from the phase separation in 
the loop binodal. This might imply that, for $L_{d^{-}} L_{o}$ phase separation, metastable nanoscopic domains (due to the stabilizing effect of the $1 \mathrm{D}$ spontaneous curvature but the destabilizing effect of a small but positive line tension) are only possible. However, the focus of this paper is on the generic properties of SHC membranes and not on a detailed microscopic model. The reduction of the line tension by adjusting the chain conformational degree of freedom of hybrid lipids and the absence of the mixing entropy cost for hybrid lipids localized at the interface between domains is a generic property of SHC membranes. It is therefore not unreasonable that biological membranes could take advantage of this physical mechanism (that we predict can result in zero or negative line tensions) to stabilize nanoscopic domains - at least for those systems in which hybrid lipids play an important role.

Since we have extensively used the analogy between hybrid lipids and surfactant at oil-water interface [13,14], it is worth noting the difference between surfactants at oil-water interfaces and the role of hybrid lipids in SHC membranes. Surfactants reduce the surface tension between oil-water interface by orienting their hydrophobic chains and hydrophilic heads towards the oil and water phases, respectively. The interactions responsible are mostly determined by the chemistry. On the other hand, hybrid lipids reduce the line tension by reconciling the chain packing incompatibility at the interfaces between domains. In this case, the interactions are physical and depend on the extent of chain ordering which is adjustable by the system. In order to decrease the free energy to a maximal extent, the chain conformational degrees of freedom of hybrid lipids may be a very efficient way to reduce the line tension to zero.

Finally, it is well known that three-body interactions among oil, water, and surfactant are responsible for the reduction of the surface tension at oil-water interfaces [16]. Since cholesterol is only modeled via an increase in the lateral pressure, the present model is essentially a model for a two-component system; there is no explicit surfactant and there are no three-body interactions between different lipid species. However, effectively there are indeed three types of lipids: hybrids whose saturated chains are disordered (in the bulk of hybrid-rich phase), hybrids whose saturated chain is ordered (near the interface between the hybrid-rich and saturated-rich phases) and saturated lipids. In other words, domains in SHC membranes are "microemulsions" due to adjustible chain order of the hybrids that varies with their position in the system. The chain order degree of freedom of the hybrids enables SHC membranes to achieve zero line tension (at least under some conditions) with only two lipid species.

The authors thank P. Pincus, L. AdDAdi, D. Andelman, R. Lipowsky, R. Ziblat, and L. Silva for helpful discussions. This research is made possible in part by the historic generosity of the Harold Perlman Family Foundation. This work was supported by the US-Israel Binational Science Foundation and an ISF converging technology grant.

\section{REFERENCES}

[1] Simons K. and Toomre D., Nat. Rev. Mol. Cell. Biol., 1 (2000) 31.

[2] Hancock J. F., Nat. Rev. Mol. Cell Biol, 7 (2006) 456.

[3] Veatch S. L. and Keller S. L., Biophys. J., 85 (2003) 3074.

[4] Veatch S. L., Soubias O., Keller S. L. and Gawrisch K., Proc. Natl. Acad. Sci. U.S.A., 104 (2007) 17650.

[5] van Meer G., Voelker D. and Feigenson G., Nat. Rev. Mol. Cell. Biol., 9 (2008) 112.

[6] van Deenen L. L. M., Pure Appl. Chem., 25 (1971) 25.

[7] de Almeida R. F. M., Loura L. M. S., Fedorov A. and Prieto M., J. Mol. Biol., 346 (2005) 1109.

[8] Feigenson G.W., Annu. Rev. Biophys. Biomol. Struct., 36 (2007) 63.

[9] Рoкоmy A., Yandek L. E., Elegbede A. I., Hinderliter A. and Almeida P. F., Biophys. J., 91 (2006) 2184.

[10] Veatch S. L. and Keller S. L., Phys. Rev. Lett., 94 (2005) 148101.

[11] Elliot R., Szleifer I. and Schick M., Phy. Rev. Lett., 96 (2006) 098101.

[12] Garbes Putzel G. and Schick M., Biophys. J., 95 (2008) 4756.

[13] Brewster R. and Safran S. A., Biophys. J., 98 (2010) L21.

[14] Brewster R., Pincus P. A. and Safran S. A., Biophys. J., 97 (2009) 1087.

[15] SAFran S. A., Statistical Thermodynamics of Surfaces Interfaces, and Membranes (Westview Press, Boulder, Col.) 2003.

[16] Gommper G. and Schick M., Phase Transitions and Critical Phenomena, edited by Domb C. and LEBowitz J. L., Vol. 16 (Academic Press, London) 1994, pp. 22-24.

[17] Yамамото T. et al., in preparation.

[18] Gelbart W. M., Ben-Shaul A. and Roux D. (Editors), Micelles, Membranes, Microemulsions, and Monolayers (Springer-Verlag, New York) 1994, pp. 1-104.

[19] Marcelja S., Nature, 241 (1973) 452.

[20] Grunen D. W. R., Biochim. Biophys. Acta, 595 (1980) 161.

[21] Helfrich W., Z. Natuforsch., 28c (1973) 693.

[22] Ou-Yang Z. C., Liu J. X. and Xie Y. Z., Geometric Methods in the Elastic Theory of Membranes in Liquid Crystal Phase (World Scientific, Singapore) 1999.

[23] De Gennes P. G. and Prost J., The Physics of Liquid Crystals (Oxford University Press, Oxford) 1993.

[24] Brochard F., Jouffroy J. and Levinson P., J. Phys. (Paris), 45 (1984) 1125.

[25] Radhakrishnan A. and McConnell H. M., Biophys. J., 77 (1999) 1507.

[26] Komura S., Shirotori H., Olmsted P. D. and Andelman D., Europhys. Lett., 67 (2004) 321.

[27] Ziblat R., Kjaer K., Leiserowitz L. and Addadi L., Angew. Chem. Int. Ed., 48 (2009) 8958. 